\title{
Die SÄZ - ein Teil der Lösung
}

\section{Bruno Kesseli}

Dr. med. et lic. phil., Chefredaktor

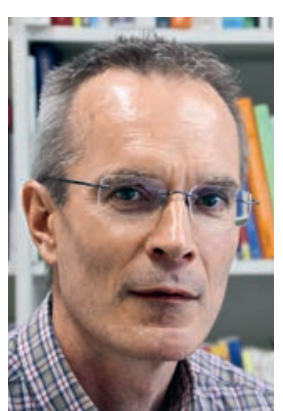

«Die Zukunft selber gestalten!»: Der Titel des ersten SÄZEditorials [1] des Jahres 2018, verfasst von FMH-Präsident Jürg Schlup, war ein Appell an die Ärztinnen und Ärzte in der Schweiz. Angesichts der grossen gesundheitspolitischen Herausforderungen rief er die Ärzteschaft dazu auf, konstruktiv zu argumentieren und Lösungen anzubieten. Entsprechende Anstrengungen trugen im Lauf des Jahres bereits erste Früchte. So konnte Zentralvorstandsmitglied Urs Stoffel im Editorial [2] der SÄZ Nr. 46 ein «überzeugtes JA der Ärzteschaft zur Tarifrevision» vermelden. Mit der Zustimmung der übrigen Tarifpartner gelang es, eine jahrelange Blockade zu überwinden und den «Durchbruch für eine gemeinsame Lösung zu schaffen».

\section{Neben der Funktion des offiziellen Organs ist die SÄZ auch eine offene Informations- und Diskussionsplattform.}

Konstruktiv argumentieren und Lösungen anbieten. Die SÄZ ist ein wichtiges Instrument in diesem Prozess. Etwas zugespitzt kann man sie durchaus als Teil der Lösung bezeichnen. Denn welche andere Plattform steht der gesamten Ärzteschaft der Schweiz - wie auch weiteren Akteuren des Gesundheitssystems - offen, um gesundheitspolitische, -ethische, -rechtliche oder -ökonomische Fragen und Probleme zu analysieren, zu diskutieren und Lösungen dafür vorzuschlagen? Zentral für dieses «Erfolgsmodell» ist aus meiner Sicht das duale System, das der SÄZ in der aktuellen Konzeption zugrunde liegt. Sie ist einerseits das offizielle Organ der FMH, deren Stellungnahmen und Positionierungen im vorderen, blau markierten Teil der SÄZ klar als solche erkennbar sind. Dass die Botschaften ankommen, belegte einmal mehr die im letzten Jahr vom Marktforschungsinstitut gfs Zürich durchgeführte repräsentative Befragung der Schweizer Ärztinnen und Ärzte [3]. Das Editorial der FMH beispielsweise erreicht rund 5000 regelmässige und 29000 gelegentliche Leserinnen und Leser. Es darf davon ausgegangen werden, dass die SÄZ auch ohne Impact Factor durchaus Impact hat.

Genauso wichtig - zumindest aus der in diesem Punkt zugegebenermassen nicht ganz unvoreingenommenen Perspektive des Chefredaktors - ist die zweite Hälfte des dualen Modells. Neben der Funktion des offiziellen Organs ist die SÄZ wie angesprochen auch eine offene Informations- und Diskussionsplattform. Unsere seit 2012 in Betrieb stehende Datenbank listet bereits über 2600 verschiedene Autoren von SÄZ-Artikeln auf, was mich auch schon zur scherzhaften Aussage verleitet hat, ich leite die wohl grösste Redaktion der Welt. Jedenfalls ist die Vielzahl an Autorinnen und Autoren, die ein enormes thematisches Spektrum abdecken und sich auch bezüglich ihrer (gesundheits-) politischen, ethischen und gesellschaftlichen Anschauungen ganz unterschiedlich positionieren, ein klares Indiz dafür, dass das duale Modell SÄZ einem Bedürfnis entspricht und funktioniert.

Zuständig dafür, dass es funktioniert, ist die SÄZRedaktion. Sie prüft und diskutiert die eingehenden Artikel. Dieses Vorgehen bietet Gewähr für breit abgestützte Entscheidungen, die sich zudem an den Autorenrichtlinien und an der Vorgabe orientieren, die gesundheitspolitische Landschaft der Schweiz und speziell die Diskussionen innerhalb der Ärzteschaft in ihrer ganzen Breite abzubilden. Ich möchte meinen Kolleginnen und Kollegen für die engagierte Arbeit, die sie - in den meisten Fällen schon über viele Jahre für die Schweizerische Ärztezeitung leisten, an dieser Stelle herzlich danken.

«Die Vielzahl an Autorinnen und Autoren ist ein klares Indiz dafür, dass das duale Modell SÄZ einem Bedürfnis entspricht und funktioniert.»

Danken möchte ich auch Ihnen, liebe Leserinnen und Leser, die Sie ja zu einem grossen Teil auch unsere Autorinnen und Autoren sind, für Ihr Interesse und Ihre Beiträge. Aus den Rückmeldungen, die ich täglich erhalte, weiss ich, dass unsere Arbeit grösstenteils geschätzt wird, auch wenn man sich gelegentlich über die SÄZ ärgert. Ich hoffe, dass dies bei dieser letzten Ausgabe des Jahres 2018 nicht der Fall sein wird. Ich wünsche Ihnen, liebe Leserin, liebe Leser, eine frohe Weihnachtszeit und einen guten Start ins neue Jahr.

Literatur

1 Schlup J. Die Zukunft selber gestalten. Schweiz Ärzteztg. 2018;99(1/2):3.

2 Stoffel U. Überzeugtes JA der Ärzteschaft zur Tarifrevision! Schweiz Ärzteztg. 2018;99(46):1601.

3 Siehe dazu: Kesseli B. Die SÄZ ist auf Kurs. Schweiz Ärzteztg. 2018;98(51/52):1758. 